\title{
Effects of a human microenvironment on the differentiation of human myoblasts
}

\author{
Matthias Catteau a , Farès Gouzi ${ }^{\mathrm{b}}$, Léo Blervaque ${ }^{\mathrm{a}}$, Emilie Passerieux ${ }^{\mathrm{a}}$, Marine Blaquière ${ }^{\mathrm{b}}$, \\ Bronia Ayoub ${ }^{b}$, François Bughin ${ }^{b}$, Jacques Mercier ${ }^{b}$, Maurice Hayot ${ }^{b}$, Pascal Pomiès ${ }^{a}{ }^{*}$ \\ a PhyMedExp, Université de Montpellier, INSERM, CNRS, 34295, Montpellier, France \\ b PhyMedExp, Université de Montpellier, INSERM, CNRS, CHU de Montpellier, 34295, Montpellier, France
}

\section{Keywords:}

COPD

Satellite cells

Myogenesis

Muscle atrophy

Cellular model

\begin{abstract}
A B S T R A T
Myogenic differentiation mechanisms are generally assessed using a murine cell line placed in low concentrations of an animal-derived serum. To more closely approximate in vivo pathophysiological conditions, recent studies have combined the use of human muscle cells with human serum. Nevertheless, the in vitro studies of the effects of a human microenvironment on the differentiation process of human myoblasts require the identification of the culture conditions that would provide an optimal and reproducible differentiation process of human muscle cells. We assessed the differentiation variability resulting from the use of human myoblasts and serums from healthy subjects by measuring the myotube diameter, fusion index and surface covered by myotubes. We showed the preserved cell-dependent variability of the differentiation response of myoblasts cultured in human serums compared to FBS. We found that using a pool of serums reduced the serum-dependent variability of the myogenic response compared to individual serums. We validated our methodology by showing the atrophying effect of pooled serums from COPD patients on healthy human myotubes. By replacing animal-derived tissues with human myoblasts and serums, and by validating the sensitivity of cultured human muscle cells to a pathological microenvironment, this human cell culture model offers a valuable tool for studying the role of the microenvironment in chronic disease.
\end{abstract}

\section{Introduction}

Most studies in the field of satellite cell differentiation are performed with standardized animal myogenic cell lines (mouse C2C12 or rat L6) cultured with animal-derived serums from horse, calf or fetal bovine. The use of human satellite cells in vitro may be a promising tool to replace animal cell lines in studies of muscle cell differentiation and regeneration, as doing so may more closely approximate in vivo pathophysiological conditions. Several research groups have thus modified their experimental parameters by using human myoblasts [1-3], a human microenvironment - i.e., the local environment of the muscle cell brought by blood flow (in vivo) or serum (in vitro) [4,5] - or both [6-8]. Nevertheless, the seeding density, differentiation durations, serum batches and cell passages are some of the factors that need to be adjusted to prevent harmful experimental heterogeneity and

\footnotetext{
* Corresponding author.

E-mail address: pascal.pomies@inserm.fr (P. Pomiès).
}

ensure the reproducibility of results [9-11]. Therefore, the biological variability in human samples must be better assessed if we want to increase the reliability of studies on in vivo muscle alteration. Biological variability is expressed at a cellular level by the heterogeneous magnitude of differentiation of human myoblasts from different subjects [12], as well as by the heterogeneous composition of different human serums compared to a controlled batch of animal serum.

Muscle differentiation and regeneration are precise and complex signaling programs that must be finely tuned to achieve the complete formation of muscle fibers [13]. Modifying the satellite cell microenvironment by various molecules impacts the differentiation process and leads to abnormalities like muscle atrophy. For example, adding hydrogen peroxide [14], metformin [15] or tumor necrosis factor-alpha [16] impairs muscle differentiation of both human and mouse $\mathrm{C} 2 \mathrm{C} 12$ myoblasts. Therefore, it is clear that a modification in the microenvironment by adding molecules is sensed by cultured muscle cells, but little is known concerning the sensitivity of human satellite cells exposed to a pathological 
microenvironment, particularly the human serum from patients with chronic disease.

In the present study, we sought to characterize the experimental parameters for an in vitro culture of human muscle cells in a human microenvironment. We therefore assessed the cell-dependent variability and the serum-dependent variability of the differentiation process in different human myoblast cultures exposed to different human serums. Then, we tested the sensitivity of our model by comparing the myogenic differentiation of healthy human myoblasts exposed to serums from healthy subjects to those from patients with chronic obstructive pulmonary disease (COPD).

\section{Materials and methods}

\subsection{Study population}

The healthy subjects were recruited on the basis of the following criteria: age between 40 and 80 years, no diagnosed disease, and low physical activity per week ( $<150$ min of moderate-to-vigorous physical activity). Age-matched COPD patients were recruited on the basis of dyspnea, chronic cough and/or sputum production, and/or a history of exposure to risk factors for the disease. Diagnosis was confirmed by forced expiratory volume in $1 \mathrm{~s}$ /forced vital capacity ratio $\left(\mathrm{FEV}_{1} / \mathrm{FVC}\right)$ lower than $70 \%$ [17]. The dyspnea severity was assessed using the modified Medical Research Council (mMRC) scale [18]. Body composition and nutritional status were evaluated with the fat-free-mass index (FFMI) and/or the body mass index (BMI) $[19,20]$. Exercise capacity and exercise tolerance were respectively reflected by the VO2peak $\left(\mathrm{VO}_{2 \mathrm{P}}\right)$ and the 6 -min walk distance (6-MWD) [21]. A written consent was obtained from all subjects (see details in Supplementary Materials and Methods).

\subsection{Myoblast isolation and purification}

As previously described [12,22], satellites cells were isolated by placing a small explant of a vastus lateralis muscle biopsy in a 35$\mathrm{mm}$ collagen-coated Petri dish covered with $6 \mathrm{mg} / \mathrm{ml}$ of Matrigel (BD Matrigel Matrix from BD Biosciences, Franklin Lakes, NJ, USA). The isolation medium was composed of DMEM (Sigma-Aldrich, St Louis, MO, USA) supplemented with 20\% FBS (Dominique Dutscher SAS, Burmath, France), 0.5\% Ultroser G (Pall Biosepra, Cergy-SaintChristophe, France) and $20 \mathrm{mM}$ Hepes (Sigma-Aldrich). Explants were cultivated for $6-8$ days in standard conditions $\left(37^{\circ} \mathrm{C} / 5 \% \mathrm{CO}_{2} /\right.$ $21 \% \mathrm{O}_{2}$ ), and outgoing cells were harvested using dispase (BD Biosciences) and grown in 100-mm collagen-coated Petri dishes with growth medium (GM; DMEM/20\% FBS/0.5\% Ultroser G). After incubation with an anti-CD56 antibody coupled with magnetic beads (BD Biosciences) for $30 \mathrm{~min}$, satellites cells were purified with a magnetic sorting system (Miltenyi Biotec, Bergisch Gladbach, Germany). The purified myoblasts (passage 1) were grown in a $100-\mathrm{mm}$ collagen-coated dish in GM and frozen at passage 2. All experiments were performed using myoblasts below passage 4 .

\subsection{Human muscle cell cultures}

Myoblasts were thawed in 100-mm Petri dishes containing GM for $48 \mathrm{~h}$. Myoblasts were then seeded in 6-well Petri plates and grown for $48 \mathrm{~h}$. Differentiation into myotubes was induced by placing the cells in differentiation medium (DM; DMEM/2\% FBS or horse serum or human healthy serum [hHS] or COPD serum) for 5 days, with a change of the medium at day 2 of the differentiation process.

\section{Results}

\subsection{Culture conditions providing optimal differentiation of human myoblasts}

In differentiation studies of primary human myoblasts, it is important to control the various factors that induce experimental variability: cell passage number [23], FBS batch [24], differentiation duration and number of seeded cells [3,14,22]. We addressed these issues by exclusively using human myoblasts below passage 4 , a single FBS batch, a standard differentiation duration of 5 days, and the induction of differentiation when the cells reached $80 \%$ confluence. Under these conditions, using a Bland-Altman plot, we assessed the reproducibility of the measures of several differentiation characteristics in the muscle cell cultures from five healthy subjects in two independent experiments (Figs. S1A-C). All the values were within the limits of agreement and a bias of less than $5 \%$ indicated no major systematic error (Figs. S1A-C). The cumulative relative frequency analysis of the myotube diameters showed no significant difference between the myotube distributions ( $p=0.26$, Fig. S1D).

To more closely approximate in vivo conditions, we assessed the differentiation characteristics of the human muscle cells in a human microenvironment by replacing the FBS with human serum from healthy subjects. We thus induced the differentiation of myoblasts from a single healthy subject, seeded at various densities, in presence of DM supplemented with a pool of serum from eight healthy subjects. We found that the 5000 cells $/ \mathrm{cm}^{2}$ condition offered the best condition for differentiation, with a high percentage of myotubes per field (88.9\%) indicating the highest number of formed myotubes per field $(54.2 \pm 7.9)$, in association with a low number of detaching myotubes $(0.6 \pm 1.3 /$ field $)$ (Table S1, Fig. S2A). Using the 5000 cells $/ \mathrm{cm}^{2}$ condition, we obtained a myotube diameter $(60 \pm 3.1 \mu \mathrm{m})$ and a fusion index $(68 \pm 3 \%)$ within the range described in previous studies $[6,8,12]$ (Fig. S2B).

\subsection{Cell-dependent variability of the differentiation process of human myoblast cultures}

Using the 5000 cells $/ \mathrm{cm}^{2}$ condition, we investigated the variability of the differentiation process by exposing the human muscle cells from seven healthy subjects (Table S2) to DM supplemented with a single hHS or FBS (Fig. 1A). The myotubes differentiated in hHS as opposed to FBS showed a higher fusion index (65.4 \pm 11.8 vs $58 \pm 10.7 \%, p=0.005$; Fig. $1 \mathrm{~B}$ ) and also tended to cover a greater surface $(39.5 \pm 7.3$ vs $33.1 \pm 6.3, p=0.058$; Fig. $1 C)$. Despite these morphological differences, the hHS preserved the intrinsic variability of the differentiation response compared to FBS, and this was reflected by equivalent coefficients of variation (C.Vs) for the fusion index (18.1 vs $18.4 \%, \mathrm{p}=0.81$; Fig. $1 \mathrm{~B}$ ) and myotube surface (18.5 vs $19.0 \%, \mathrm{p}=0.72$; Fig. $1 \mathrm{C}$ ). Under the same experimental conditions, hHS tended to increase the myotube diameter by $24 \%$ $(58 \pm 18$ vs $47 \pm 18 \mu \mathrm{m}, \mathrm{p}=0.064$, Fig. 1D) without affecting the C.V of the myotube cultures ( 30.5 vs $38.0 \%, p=0.99$; Fig. 1D). However, the linear mixed model showed that hHS increased the mean myotube diameter ( $p<0.001$, Fig. S3). Indeed, the use of hHS induced a shift in the distribution curve toward higher myotube diameters (Fig. 1E). Furthermore, cumulative frequency analysis of the myotube diameters showed a significant difference between FBS and hHS ( $p<0.001$ ), with $50 \%$ of the myotubes differentiated in FBS measuring less than $37.7 \mu \mathrm{m}$, whereas those in hHS had a diameter under $46.5 \mu \mathrm{m}$ (Fig. 1F). 


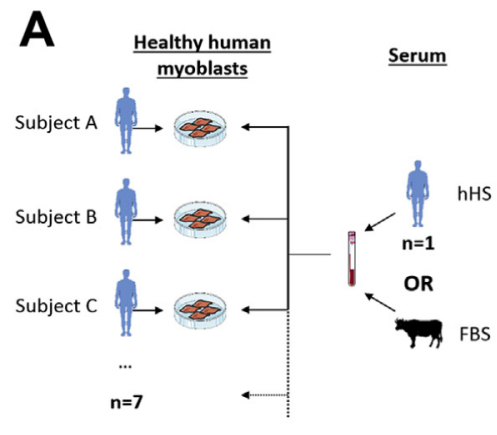

$\mathbf{E}$

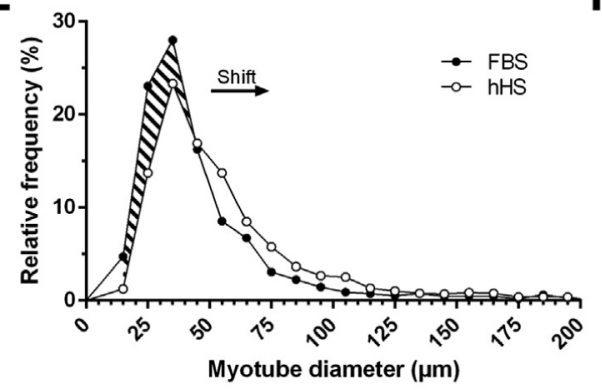

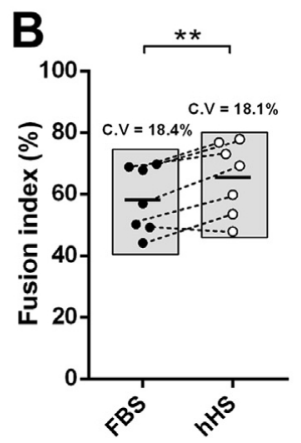
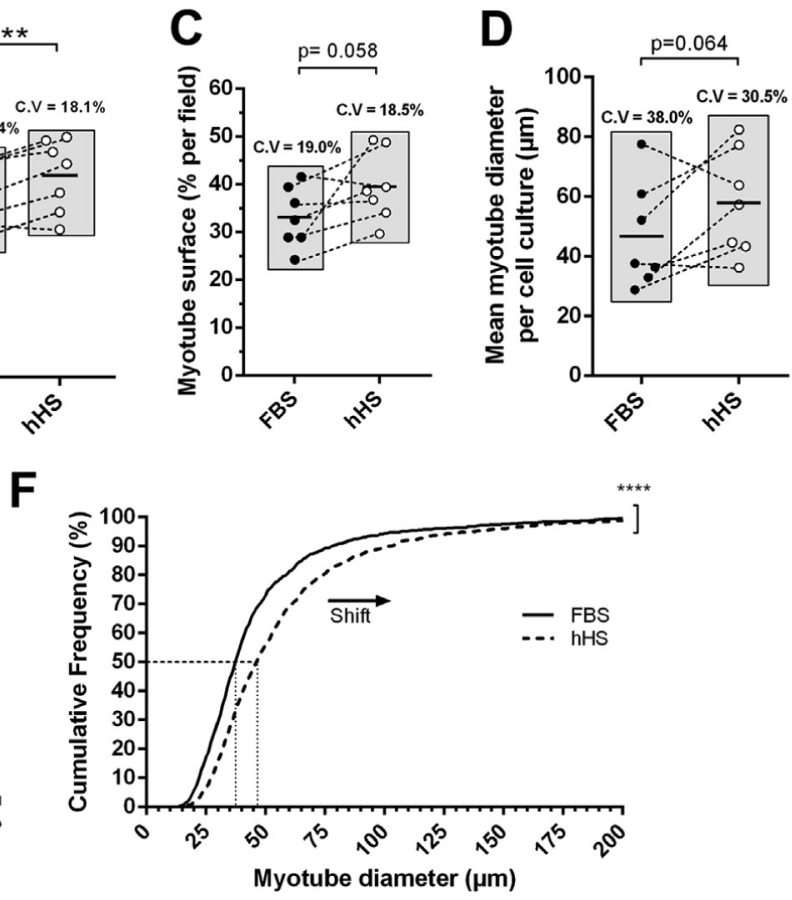

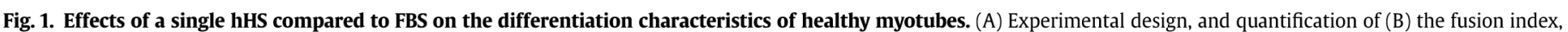

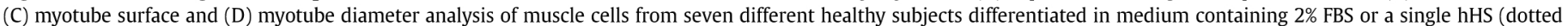

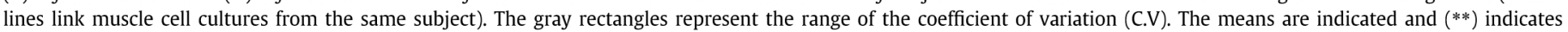

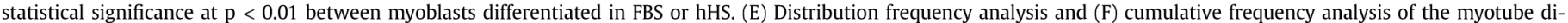

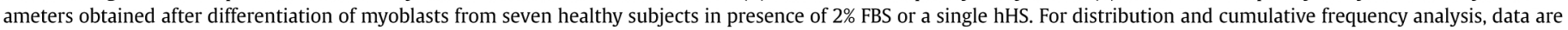
expressed in percentage of all myotube diameters per condition and (****) indicates statistical significance at p $<0.0001$ with Kolmogorov-Smirnov's distribution test.

\subsection{Serum-dependent variability of the differentiation process of a human myoblast culture}

We also investigated the variability of several human serums to induce myogenic differentiation by differentiating the myoblasts from a single healthy subject in presence of eight individual hHS (Fig. 2A). Compared with the reference values obtained with FBS, the myotubes exposed to individual hHS showed an increase in myotube diameter $(+55 \%, \mathrm{p}<0.001$; Fig. $2 \mathrm{~B})$, fusion index $(+25 \%$, $\mathrm{p}<0.0001$; Fig. $2 \mathrm{C}$ ) and covered surface (+43\%, $\mathrm{p}<0.01$; Fig. 2D), with C.Vs of $17.6 \%, 6.9 \%$ and $17.3 \%$, respectively.

\subsection{Differentiation response of human myoblasts exposed to individual human serums or pooled serums}

To reduce the serum-dependent variability, we assessed the effects of pooled human serums on the differentiation characteristics of the myoblasts, as performed by other groups [4,7]. We compared the effects of eight individual hHS to the effects of a pool of the same eight serums (Fig. 3A). Use of the pooled serums reduced the C.Vs of each measurement (17.6-2.9\% for myotube diameter, 6.9 to $1.7 \%$ for fusion index, and 17.3 to $0.6 \%$ for covered surface; Table S3, Fig. 3B-D) without affecting the ability to induce myotube differentiation. Indeed, both pooled and individual serums retained an equivalent ability to induce fusion with similar trophic effects on myotube diameter (61 vs $69 \mu \mathrm{m}, \mathrm{p}=0.09$; Fig. 3B), fusion index (71.1 vs $74.5 \%, p=0.10$; Fig. $3 C$ ), and surface covered by myotubes (36.0 vs $33.3 \%, \mathrm{p}=0.24$; Fig. 3D).

As the pooled serums retained biological effects similar to those of individual serums, we compared the differentiation characteristics of human myoblast cultures exposed to pooled hHS or FBS (Fig. 3E). Distribution frequency analysis of the myotube diameters
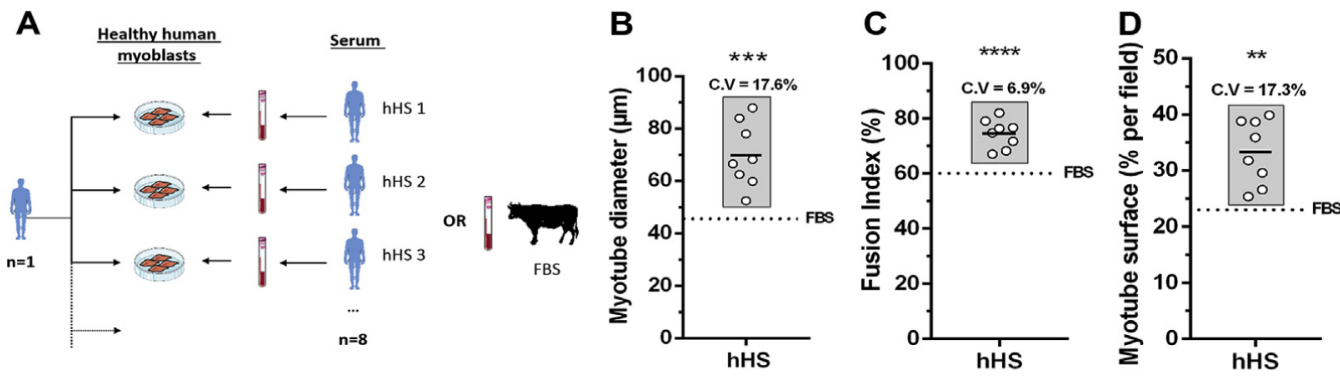

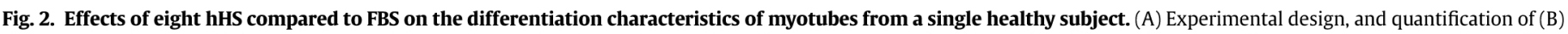

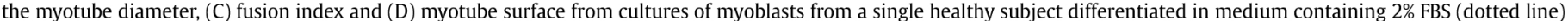

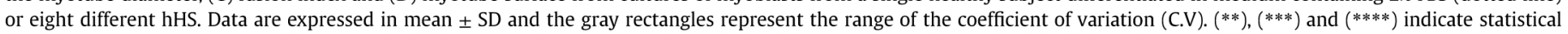
significance at $\mathrm{p}<0.01, \mathrm{p}<0.001$ and $\mathrm{p}<0.0001$ respectively, between values obtained for myotubes differentiated in FBS and individual hHS. 
A

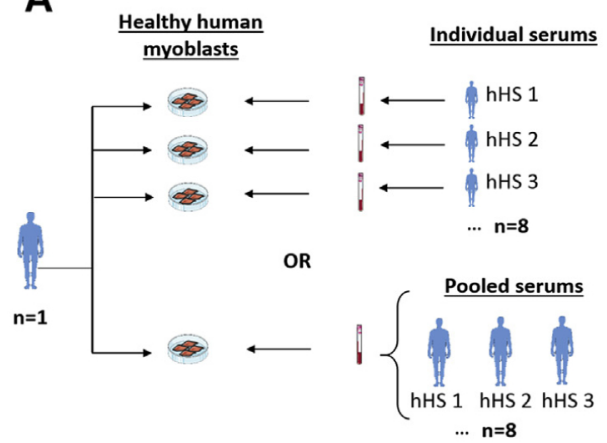

E Healthy human

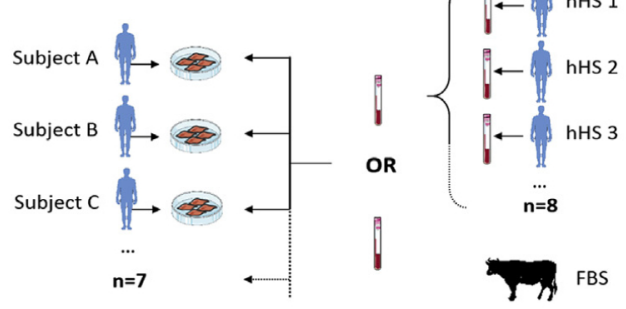

B
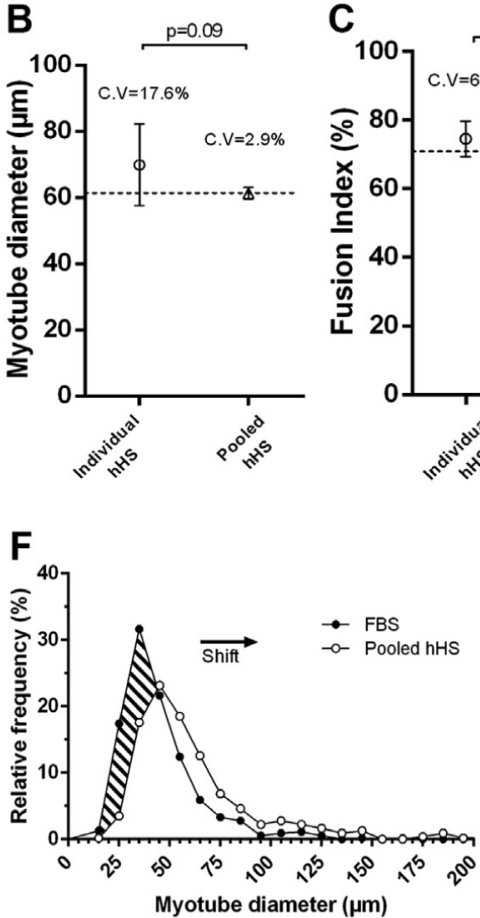
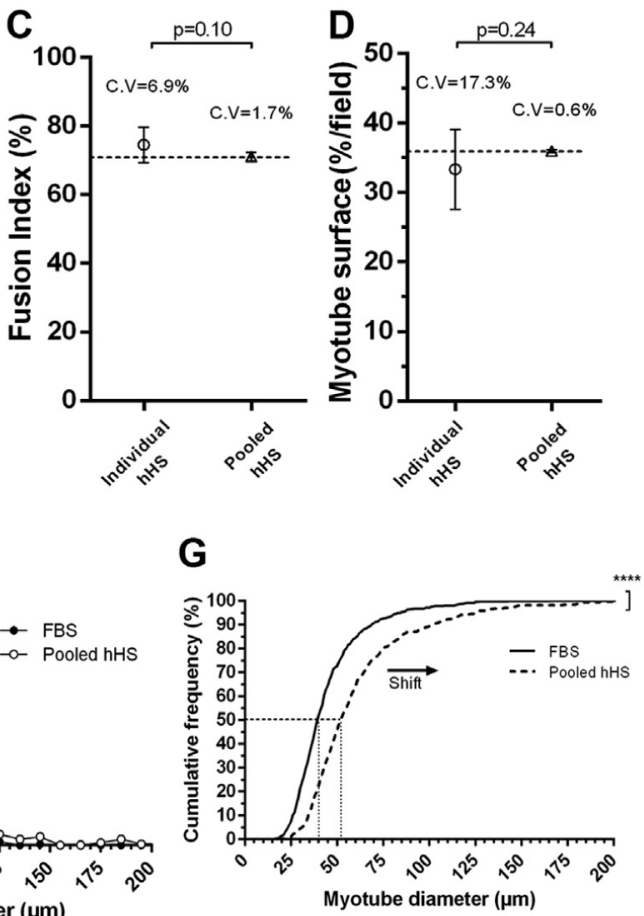

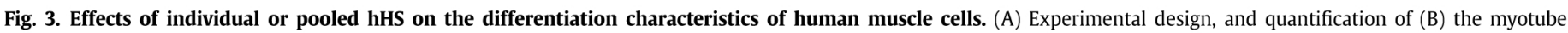

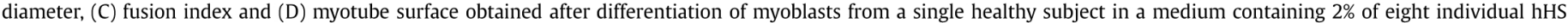

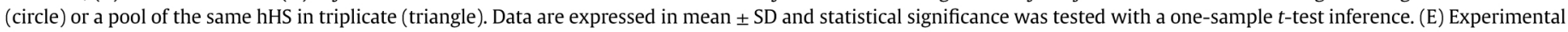

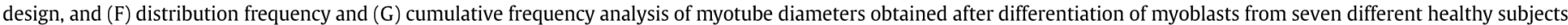

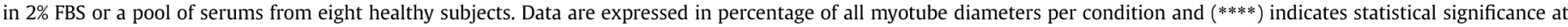
$\mathrm{p}<0.0001$ with Kolmogorov-Smirnov's distribution test.

showed that the pooled hHS, compared to FBS, induced a shift toward higher myotube diameters (Fig. 3F), similar to that observed with individual hHS in Fig. 1E. Furthermore, the cumulative frequency curves indicated a significant difference between hHS and FBS ( $p<0.0001$ ), with $50 \%$ of the myotubes differentiated in FBS measuring less than $39.8 \mu \mathrm{m}$ against $57.2 \mu \mathrm{m}$ in hHS (Fig. 3G).

\subsection{Sensitivity of healthy human myoblasts to serum from COPD patients}

To assess the sensitivity of healthy human muscle cells to pathological serum, we compared the effects of pooled serums from COPD patients to those from healthy subjects on their differentiation characteristics. The subject characteristics are described in Table S4. We induced the differentiation of myoblasts from seven healthy subjects with pooled serums from eight healthy subjects or eight COPD patients (Fig. 4A). Representative images of the cultured myotubes are shown in Fig. 4B. While no difference was detected for the fusion index $(60.5 \pm 10.3$ vs $59.7 \pm 10.9 \%, p=0.60$; Fig. $4 \mathrm{C})$, we observed a significant decrease in the surface covered by the myotubes exposed to the COPD serums $(36.0 \pm 4.3$ vs $33.0 \pm 3.9 \%$, $\mathrm{p}<0.05$; Fig. 4D). Concomitantly, the COPD serums tended to reduce the myotube diameter $(53 \pm 14$ vs $49 \pm 12 \mu \mathrm{m}, \mathrm{p}=0.09$; Fig. 4E). A linear mixed model also indicated this tendency, with a significant reduction in the mean myotube diameter $(\mathrm{p}<0.01$; Fig. S4). Distribution frequency analysis of the myotube diameters showed that the COPD serums induced a shift toward thinner myotubes compared to hHS (Fig. 4F). In addition, cumulative frequency analysis showed a significant difference in the myotube diameter distribution ( $p<0.001$ ), with $50 \%$ of the myotubes differentiated in COPD serums being $8 \%$ thinner than those in hHS (40.5 vs $43.8 \mu \mathrm{m}$, Fig. 4G).

\section{Discussion}

Given the heterogeneity of cell culture conditions [6-8,12,22,25], we sought to define experimental procedures for culturing human muscle cells to ensure the reproducibility of measurements by our group and other teams. We found that 5000 cells $/ \mathrm{cm}^{2}$ provided the highest number of myotubes, with a limited number of cell detachments (Table S1). Under these conditions, the fusion index and the myotube surface/diameter values were in the range of those described by others $[6,8,22,26]$.

As our primary human myoblasts were isolated from different healthy subjects, we investigated the intrinsic variability of each culture. In these experiments, no significant difference in the C.Vs of the myogenic indicators suggested that human serum, compared to FBS, was able to preserve the variability of the response of each myoblast culture (Fig. 1B-D). However, although variability was preserved, the human serum seemed to modify the differentiation dynamics (Fig. 1B), with increased myotube surface (Fig. 1C) and diameter (Fig. 1D). Using a linear mixed model taking into account the value dependency related to repetitive observations (myotube diameter) performed within the same experimental object (myotubes from the same subject) (Fig. S3) [27,28], we confirmed the tendency of human serum to increase myotube diameter (Fig. 1E-F). While industrial serums (FBS, calf serum, horse serum) commonly used for in vitro muscle cultures condition cellular processes such as proliferation, differentiation, fusion and protein synthesis [29], we confirmed that serums from different healthy subjects are also able to modify myogenesis (Fig. 2).

To better approximate a representative serum composition for a global population, we adapted our procedure by pooling different human serums, as previously described in the field $[2,7,8]$. This enabled us to optimize the volume of serum used for each 
A

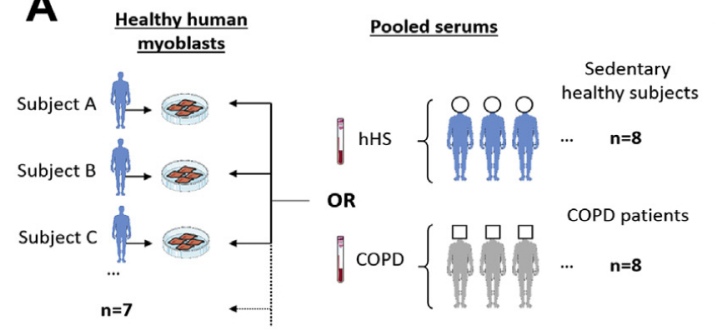

B

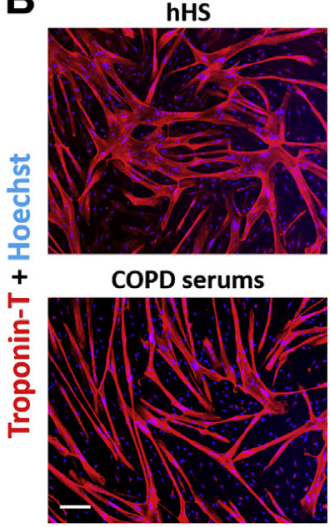

C

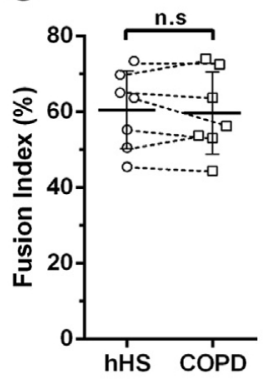

D

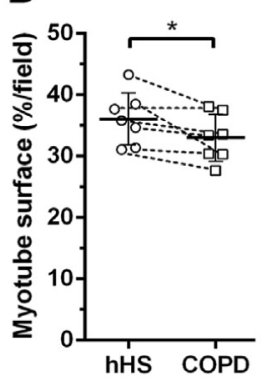

E

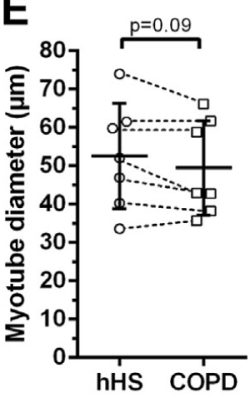

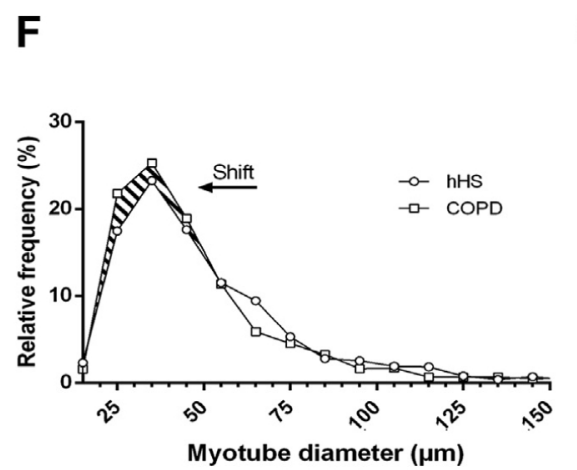

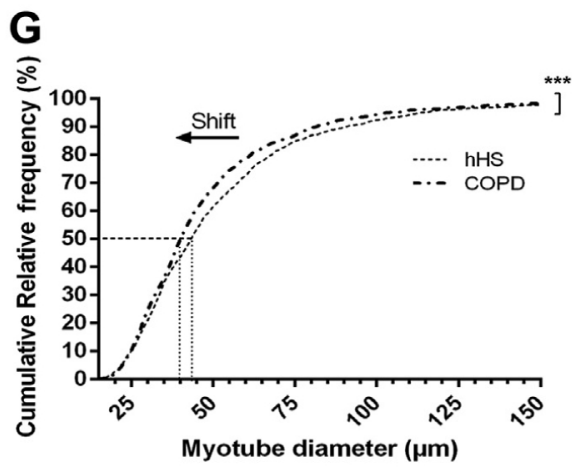

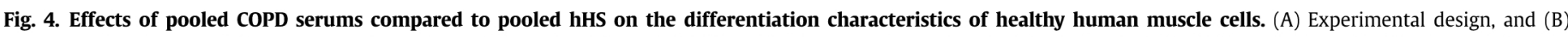

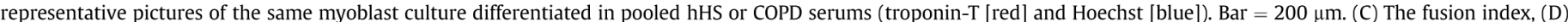

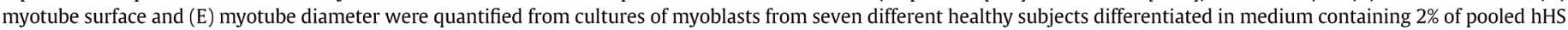

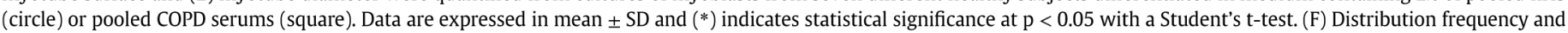

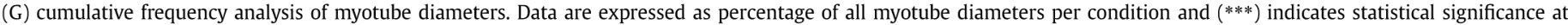

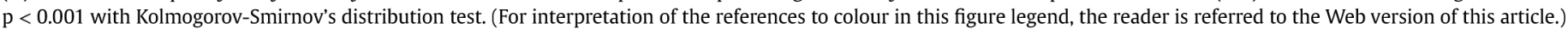

experiment and reduce the serum-dependent variability without modifying the biological effects (Fig. 3A-D). The trophic effect of pooled human serums on differentiation was confirmed, compared to FBS (Fig. 3E-G).

The composition of the microenvironment regulates many physiological processes, and its implication in the modulation of muscle atrophy in chronic disease/aging is a complex issue that is still debated. Recently, it has been shown that aging imposes several changes on the circulating microenvironment, impacting processes such as neurogenesis, hepatogenesis and muscle regeneration [30]. More specifically in COPD, Barnes and Celli hypothesized that the skeletal muscle atrophy occurring in COPD patients is partly due to a deleterious systemic microenvironment coming from the "spill-over" of inflammatory molecules from the lungs into the blood circulation [31]. Indeed, studies have shown a sustained increase in inflammatory cells and molecules in the blood of COPD patients [32-34], correlating with sarcopenia [35]. In the present study, we offer new experimental clues about this process. Although the COPD severity of the patients was moderate, our new methodology was sensitive enough to reveal that the composition of the COPD serums had deleterious effects on healthy myotubes, inducing a higher proportion of thin myotubes to the detriment of intermediate and large myotubes (Fig. 4). Unfortunately, without the dosage of inflammatory molecules, we cannot definitively conclude on the inflammatory state of the COPD serums used in our study, and further experiments are now needed.

The in vitro methodology described here enabled us to study the effects of a homologous microenvironment on the differentiation process of human muscle cells. We highlighted the importance of assessing both cell- and serum-variability, which is a crucial prerequisite for in vitro experiments with human tissues. Furthermore, we showed that pooled human serums retain the biological effect of individual serums. Last, we validated our methodology by providing experimental clues of the "spill-over" mechanism by which deleterious molecules from the lung may contribute to the skeletal muscle atrophy occurring in COPD patients.

\section{Declaration of competing interest}

The authors declare that they have no known competing financial interests or personal relationships that could have appeared to influence the work reported in this paper.

\section{Acknowledgements}

We acknowledge the 5-Santé Group (Osséja, France) and the research group of JP Cristol (PhyMedExp, Montpellier, France) for their support in obtaining human samples. This study was supported by joint grants from the French Ministry of Public Health (PHRCI, AREB-1) and the Montpellier University Hospital, and the APARD (Montpellier, France).

\section{References}

[1] C.-H. Zhu, V. Mouly, R.N. Cooper, K. Mamchaoui, A. Bigot, J.W. Shay, J.P. Di Santo, G.S. Butler-Browne, W.E. Wright, Cellular senescence in human myoblasts is overcome by human telomerase reverse transcriptase and cyclindependent kinase 4: consequences in aging muscle and therapeutic strategies for muscular dystrophies, Aging Cell 6 (2007) 515-523, https://doi.org/ 10.1111/j.1474-9726.2007.00306.x.

[2] S. Chanon, B. Chazarin, B. Toubhans, C. Durand, I. Chery, M. Robert, A. Vieille- 
Marchiset, J.E. Swenson, A. Zedrosser, A.L. Evans, S. Brunberg, J.M. Arnemo, G. Gauquelin-Koch, K.B. Storey, C. Simon, S. Blanc, F. Bertile, E. Lefai, Proteolysis inhibition by hibernating bear serum leads to increased protein content in human muscle cells, Sci. Rep. 8 (2018), https://doi.org/10.1038/s41598018-23891-5.

[3] F. Gouzi, M. Blaquière, M. Catteau, F. Bughin, J. Maury, E. Passerieux, B. Ayoub, J. Mercier, M. Hayot, P. Pomiès, Oxidative stress regulates autophagy in cultured muscle cells of patients with chronic obstructive pulmonary disease, J. Cell. Physiol. (2018), https://doi.org/10.1002/jcp.26868.

[4] H.W. van Hees, W.--.M. Schellekens, M. Linkels, F. Leenders, J. Zoll, R. Donders, P.R. Dekhuijzen, J.G. van der Hoeven, L.M. Heunks, Plasma from septic shock patients induces loss of muscle protein, Crit. Care 15 (2011) R233.

[5] I. Kalampouka, A. van Bekhoven, B.T. Elliott, Differing effects of younger and older human plasma on C2C12 myocytes in vitro, Front. Physiol. 9 (2018), https://doi.org/10.3389/fphys.2018.00152.

[6] T. George, C.P. Velloso, M. Alsharidah, N.R. Lazarus, S.D.R. Harridge, Sera from young and older humans equally sustain proliferation and differentiation of human myoblasts, Exp. Gerontol. 45 (2010) 875-881, https://doi.org/10.1016/ j.exger.2010.07.006.

7] K.L. Corrick, M.J. Stec, E.K. Merritt, S.T. Windham, S.J. Thomas, J.M. Cross, M.M. Bamman, Serum from human burn victims impairs myogenesis and protein synthesis in primary myoblasts, Front. Physiol. 6 (2015), https:// doi.org/10.3389/fphys.2015.00184.

[8] D. Vitucci, E. Imperlini, R. Arcone, A. Alfieri, A. Canciello, L. Russomando, D. Martone, A. Cola, G. Labruna, S. Orrù, Serum from differently exercised subjects induces myogenic differentiation in LHCN-M2 human myoblasts, J. Sports Sci. 36 (2018) 1630-1639.

[9] M.A. Lawson, P.P. Purslow, Differentiation of myoblasts in serum-free media: effects of modified media are cell line-specific, Cells Tissues Organs 167 (2000) $130-137$.

[10] J. van der Valk, D. Mellor, R. Brands, R. Fischer, F. Gruber, G. Gstraunthaler, L. Hellebrekers, J. Hyllner, F.H. Jonker, P. Prieto, M. Thalen, V. Baumans, The humane collection of fetal bovine serum and possibilities for serum-free cell and tissue culture, Toxicol. Vitro 18 (2004) 1-12, https://doi.org/10.1016/ j.tiv.2003.08.009.

[11] M. Baker, Reproducibility: Respect Your Cells!, Nature Publishing Group 2016.

[12] P. Pomiès, J. Rodriguez, M. Blaquière, S. Sedraoui, F. Gouzi, G. Carnac, D. Laoudj-Chenivesse, J. Mercier, C. Préfaut, M. Hayot, Reduced myotube diameter, atrophic signalling and elevated oxidative stress in cultured satellite cells from COPD patients, J. Cell Mol. Med. 19 (2015) 175-186, https://doi.org $10.1111 / \mathrm{jcmm} .12390$.

13] T. Pietrangelo, C. Puglielli, R. Mancinelli, S. Beccafico, G. Fanò, S. Fulle, Mo lecular basis of the myogenic profile of aged human skeletal muscle satellite cells during differentiation, Exp. Gerontol. 44 (2009) 523-531, https://doi.org/ 10.1016/j.exger.2009.05.002.

[14] P. Pomiès, M. Blaquière, J. Maury, J. Mercier, F. Gouzi, M. Hayot, Involvemen of the FoxO1/MuRF1/Atrogin-1 signaling pathway in the oxidative stressinduced atrophy of cultured chronic obstructive pulmonary disease myotubes, PloS One 11 (2016), e0160092.

[15] T. Pavlidou, M. Rosina, C. Fuoco, G. Gerini, C. Gargioli, L. Castagnoli, G. Cesareni, Regulation of myoblast differentiation by metabolic perturbations induced by metformin, PloS One 12 (2017), e0182475.

[16] M. Podbregar, M. Lainscak, O. Prelovsek, T. Mars, Cytokine response of cultured skeletal muscle cells stimulated with proinflammatory factors depends on differentiation stage, Sci. World J. (2013) 1-8, https://doi.org/ $10.1155 / 2013 / 617170,2013$.

[17] C.F. Vogelmeier, G.J. Criner, F.J. Martinez, A. Anzueto, P.J. Barnes, J. Bourbeau, B.R. Celli, R. Chen, M. Decramer, L.M. Fabbri, P. Frith, D.M.G. Halpin, M.V. López Varela, M. Nishimura, N. Roche, R. Rodriguez-Roisin, D.D. Sin, D. Singh, R. Stockley, J. Vestbo, J.A. Wedzicha, A. Agustí, Global strategy for the diagnosis, management, and prevention of chronic obstructive lung disease 2017 report. gold executive summary, Am. J. Respir. Crit. Care Med. 195 (2017) 557-582, https://doi.org/10.1164/rccm.201701-0218PP.

[18] T. Perez, P.R. Burgel, J.L. Paillasseur, D. Caillaud, G. Deslée, P. Chanez, N. Roche, Modified medical research council scale vs baseline dyspnea index to evaluate dyspnea in chronic obstructive pulmonary disease, Int. J. Chronic Obstr. Pulm. Dis. (2015) 1663, https://doi.org/10.2147/COPD.S82408.

[19] F.Q. Nuttall, Body mass index: obesity, BMI, and Health, Nutr. Today 50 (2015) 117-128, https://doi.org/10.1097/NT.0000000000000092.

[20] K.A. Kudsk, A. Munoz-del-Rio, R.A. Busch, C.E. Kight, D.A. Schoeller, Stratification of fat-free mass index percentiles for body composition based on national Health and nutrition examination survey III bioelectric impedance data, J. Parenter. Enteral Nutr. 41 (2017) 249-257, https://oi.org/10.1177/ 0148607115592672.

[21] B. Celli, K. Tetzlaff, G. Criner, M.I. Polkey, F. Sciurba, R. Casaburi, R. Tal-Singer A. Kawata, D. Merrill, S. Rennard, The 6-minute-walk distance test as a chronic obstructive pulmonary disease stratification tool. Insights from the COPD biomarker qualification consortium, Am. J. Respir. Crit. Care Med. 194 (2016) 1483-1493, https://doi.org/10.1164/rccm.201508-16530C.

[22] M. Barro, G. Carnac, S. Flavier, J. Mercier, Y. Vassetzky, D. Laoudj-Chenivesse, Myoblasts from affected and non-affected FSHD muscles exhibit morphological differentiation defects, J. Cell Mol. Med. 14 (2010) 275-289, https: doi.org/10.1111/j.1582-4934.2008.00368.x

[23] K. Kwist, W.C. Bridges, K.J.L. Burg, The effect of cell passage number on osteogenic and adipogenic characteristics of D1 cells, Cytotechnology 68 (2016) 1661-1667, https://doi.org/10.1007/s10616-015-9883-8.

[24] A. Khodabukus, K. Baar, The effect of serum origin on tissue engineered skeletal muscle function: serum and engineered muscle function. J. Cell Biochem. 115 (2014) 2198-2207, https://doi.org/10.1002/jcb.24938.

[25] E. Barreiro, E. Puig-Vilanova, A. Salazar-Degracia, S. Pascual-Guardia, C. Casadevall, J. Gea, The phosphodiesterase-4 inhibitor roflumilast reverts proteolysis in skeletal muscle cells of patients with COPD cachexia, J. Appl. Physiol. $125 \quad$ (2018) 287-303, https://doi.org/10.1152/ japplphysiol.00798.2017.

[26] D.P. Millay, J.R. O'Rourke, L.B. Sutherland, S. Bezprozvannaya, J.M. Shelton, R. Bassel-Duby, E.N. Olson, Myomaker is a membrane activator of myoblast fusion and muscle formation, Nature 499 (2013) 301-305, https://doi.org/ 10.1038 /nature 12343

[27] E. Aarts, M. Verhage, J.V. Veenvliet, C.V. Dolan, S. van der Sluis, A solution to dependency: using multilevel analysis to accommodate nested data, Nat. Neurosci. 17 (2014) 491-496, https://doi.org/10.1038/nn.3648.

[28] T.V. Pollet, G. Stulp, S.P. Henzi, L. Barrett, Taking the aggravation out of data aggregation: a conceptual guide to dealing with statistical issues related to the pooling of individual-level observational data: data aggregation and the ecological fallacy, Am. J. Primatol. 77 (2015) 727-740, https://doi.org 10.1002/ajp.22405

[29] A. Saini, E. Rullman, M. Lilja, M. Mandić, M. Melin, K. Olsson, T. Gustafsson, Asymmetric cellular responses in primary human myoblasts using sera of different origin and specification, PloS One 13 (2018), e0192384, https:/ doi.org/10.1371/journal.pone.0192384.

[30] I.M. Conboy, M.J. Conboy, J. Rebo, Systemic problems: a perspective on stem cell aging and rejuvenation, Aging 7 (2015) 754.

[31] P.J. Barnes, B.R. Celli, Systemic manifestations and comorbidities of COPD, Eur Respir. J. 33 (2009) 1165-1185, https://doi.org/10.1183/09031936.00128008.

[32] A. Agustí, LD. Edwards, S.I. Rennard, W. MacNee, R. Tal-Singer, B.E. Miller, J. Vestbo, D.A. Lomas, P.M.A. Calverley, E. Wouters, C. Crim, J.C. Yates, E.K. Silverman, H.O. Coxson, P. Bakke, R.J. Mayer, B. Celli, For the evaluation of COPD longitudinally to identify predictive surrogate endpoints (eclipse) investigators, persistent systemic inflammation is associated with poor clinica outcomes in COPD: a novel phenotype, PloS One 7 (2012), e37483, https:// doi.org/10.1371/journal.pone.0037483.

[33] Pinto-Plata, C. Casanova, H. Müllerova, J.P. de Torres, H. Corado, N. Varo, E. Cordoba, S. Zeineldine, H. Paz, R. Baz, Inflammatory and Repair Serum Biomarker Pattern, Association to clinical outcomes in COPD, 2012.

[34] R.L. Zemans, S. Jacobson, J. Keene, K. Kechris, B.E. Miller, R. Tal-Singer, R.P. Bowler, Multiple biomarkers predict disease severity, progression and mortality in COPD, Respir. Res. 18 (2017), https://doi.org/10.1186/s12931017-0597-7.

[35] M.K. Byun, E.N. Cho, J. Chang, C.M. Ahn, H.J. Kim, Sarcopenia correlates with systemic inflammation in COPD, Int. J. Chronic Obstr. Pulm. Dis. 12 (2017) 669-675, https://doi.org/10.2147/COPD.S130790. 